\title{
Studies of Diluted Magnetic Semiconductor $\mathrm{Sn}_{1-x-y-z} \mathrm{Ge}_{x} \mathrm{Mn}_{y} \mathrm{Gd}_{z} \mathrm{Te}$
}

\author{
V.I. Sichkovskyi ${ }^{a}$, M.V. Radchenko ${ }^{a}$, G.V. Lashkarev ${ }^{a} *$, V.E. Slyn'Ko ${ }^{a}$, E.I. Slyn'KO ${ }^{a}$,

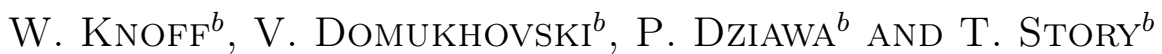 \\ ${ }^{a}$ Institute for Problems of Material Science, National Academy of Sciences of Ukraine \\ 3 Krzhizhanovskogo str., Kyiv, Ukraine \\ ${ }^{b}$ Institute of Physics, Polish Academy of Sciences, al. Lotnikow 32/46, 02-668 Warsaw, Poland \\ Magnetization, anomalous Hall effect, thermoelectric power, magnetoresistance, and resistivity of \\ $\mathrm{Sn}_{1-x-y-z} \mathrm{Ge}_{x} \mathrm{Mn}_{y} \mathrm{Gd}_{z} \mathrm{Te}(x=0.039 \div 0.597, y=0.077 \div 0.125, z=0.0014 \div 0.028)$ mixed crystals were \\ studied over the temperature range $4.2-300 \mathrm{~K}$. The ferromagnetic order with Curie temperature $18-24 \mathrm{~K}$ was \\ revealed.
}

PACS numbers: 72.20.My, 75.50.Pp, 72.80.Ga

\section{Introduction}

The family of IV-VI semiconductors notices attention due to possibility of its wide applications in different fields of electronics $[1,2]$. The special interest is attracted by diluted magnetic semiconductors (DMSs) based on IV-VI host matrices doped with $3 d$ and $4 f$ transition metals [3]. These DMSs exhibit ferromagnetic (FM) ordering caused by indirect exchange interaction of the magnetic ions via the hole gas $[4,5]$. Moreover, IV-VI semiconductors are characterized by phase transformations of ferroelectric type. At present, the searches for DMSs with high Curie temperature for spintronics applications are still in progress. It is interesting to study the effect of the simultaneous presence of two magnetic ions types in IV-VI semiconductor host on magnetic properties of resulting DMS.

\section{Experimental results and discussion}

$\mathrm{Sn}_{1-x-y-z} \mathrm{Ge}_{x} \mathrm{Mn}_{y} \mathrm{Gd}_{z} \mathrm{Te}(x=0.039 \div 0.597 ; y=$ $0.077 \div 0.125 ; z=0.0014 \div 0.028)$ bulk crystals were grown by modified Bridgman method. X-ray diffraction studies showed that these DMS crystallize in the $\mathrm{NaCl}$ crystal structure. The chemical composition was determined by X-ray fluorescent analysis, which displayed that the content of $\mathrm{Mn}$ and $\mathrm{Gd}$ changes opposite one another along the ingot. Multimeter Keithley 2700/E with data acquisition system was used for transport measurements. Magnetic properties were investigated using a SQUID magnetometer over the temperature range $5-100 \mathrm{~K}$.

The SQUID magnetometry studies of magnetization $M$ of $\mathrm{Sn}_{1-x-y-z} \mathrm{Ge}_{x} \mathrm{Mn}_{y} \mathrm{Gd}_{z}$ Te crystals (typical result is

* corresponding author; e-mail: georgl@isp.kiev.ua shown in Fig. 1) have revealed the FM transition with the Curie temperature $T_{\mathrm{C}}=18 \div 24 \mathrm{~K}$. The latter decreases with the increase of $\mathrm{Gd}$ content. It should be noted that $M(T)$ in zero-field-cooled and field-cooled regime showed a difference only for samples with high Mn content opposite to $\mathrm{Ge}_{1-x-y} \mathrm{Sn}_{x} \mathrm{Mn}_{y} \mathrm{Te}$ [6]. The $M(H)$ dependence (inset to Fig. 1) was not measured below the Curie temperature because of huge magnetic signal.

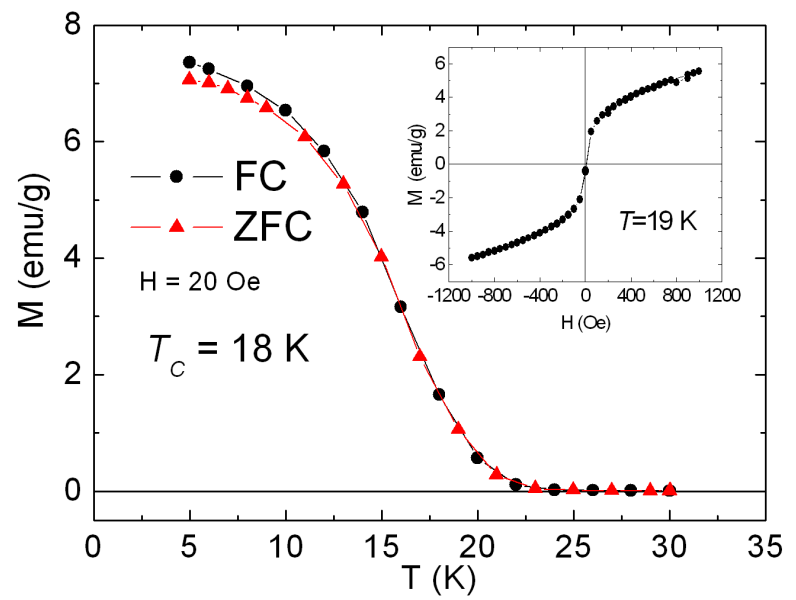

Fig. 1. Temperature dependence of magnetization of $\mathrm{Sn}_{1-x-y-z} \mathrm{Ge}_{x} \mathrm{Mn}_{y} \mathrm{Gd}_{z} \mathrm{Te}(x=0.227, y=0.088$, $z=0.0066)$. The inset shows the $M(H)$ dependence at $T=19 \mathrm{~K}$.

The transport measurements revealed semimetallic (typical of degenerated semiconductors) $p$-type conductivity for all samples. A weak feature on resistivity $\rho(T)$ dependence near $T_{\mathrm{C}}$ was observed (Fig. 2). For the samples with low Ge content a kink on $\rho(T)$ dependence at 
$T=165-175 \mathrm{~K}$ was discovered. The latter coincides with a bend on temperature dependence of thermopower $\alpha(T)$ (Fig. 2), which testifies to phase transition to cubic phase at temperature increase. Negative magnetoresistance of a very small magnitude $(0.2 \%$ at $H=6.5 \mathrm{kOe}, T=4.2 \mathrm{~K})$ appeared below $40 \mathrm{~K}$. Its small value can be explained by dominant role of spin-independent scattering processes due to high concentration of metal vacancies in IV-VI semiconductors.

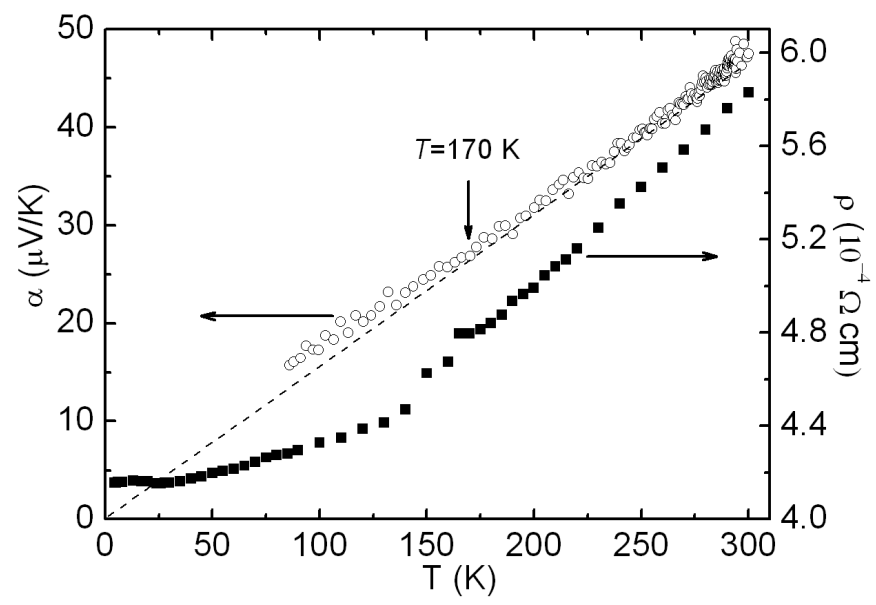

Fig. 2. Resistivity and thermopower as a function of temperature measured for $\mathrm{Sn}_{1-x-y-z} \mathrm{Ge}_{x} \mathrm{Mn}_{y} \mathrm{Gd}_{z} \mathrm{Te}$ $(x=0.039, y=0.077, z=0.028)$ sample. The vertical arrow indicates a bend on $\alpha(T)$ dependence at $T=170 \mathrm{~K}$. The dashed line is guide to the eyes.

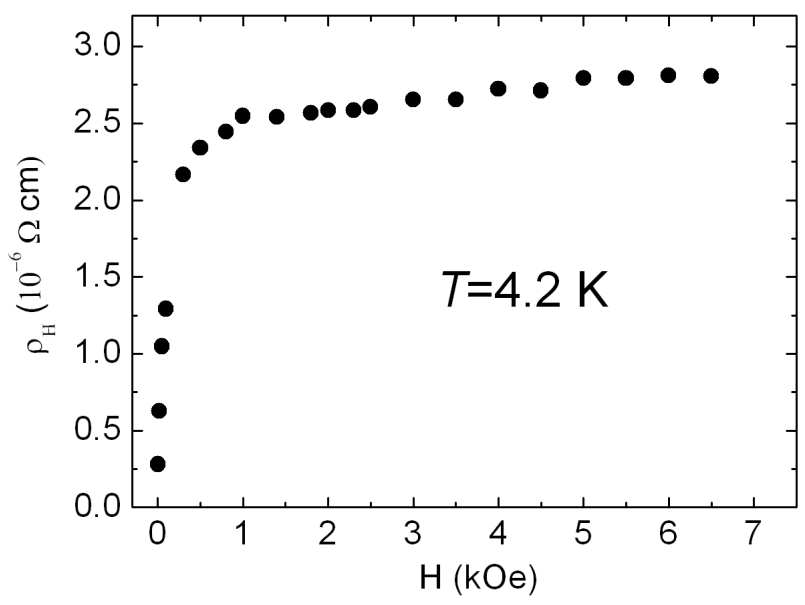

Fig. 3. The magnetic field dependence on the Hall resistivity at $T=4.2 \mathrm{~K}$ for $\mathrm{Sn}_{1-x-y-z} \mathrm{Ge}_{x} \mathrm{Mn}_{y} \mathrm{Gd}_{z} \mathrm{Te}$ $(x=0.227, y=0.088, z=0.0066)$ sample.

The dependence of Hall resistivity $\rho_{\mathrm{H}}$ versus magnetic field measured at $T=4.2 \mathrm{~K}$ displayed large contribu- tion of anomalous Hall effect proportional to magnetic moment (Fig. 3), which is another proof of FM state in $\mathrm{Sn}_{1-x-y-z} \mathrm{Ge}_{x} \mathrm{Mn}_{y} \mathrm{Gd}_{z} \mathrm{Te}$ crystals. At $H>1 \mathrm{kOe}$ weak increase in $\rho_{\mathrm{H}}$ due to input of normal Hall effect was observed. Calculated hole concentration and hole mobility are about $8.5 \times 10^{21} \mathrm{~cm}^{-3}$ and $\mu<30 \mathrm{~cm}^{2} \mathrm{~V}^{-1} \mathrm{~s}^{-1}$, respectively.

\section{Conclusions}

Single crystals $\mathrm{Sn}_{1-x-y-z} \mathrm{Ge}_{x} \mathrm{Mn}_{y} \mathrm{Gd}_{z} \mathrm{Te}$ undergo transition to ferromagnetic state with the Curie temperature in the range 18-24 K. Nevertheless, the superparamagnetic behavior of magnetic moment testifies to cluster nature of this DMS. Doping with gadolinium significantly increases the magnetic moment of the crystal at low temperatures in comparison with the Mn-based IV-VI DMSs but does not lead to the Curie temperature increase. Spin-glass-like behavior of investigated samples only for the highest manganese content was revealed unlike the $\mathrm{Ge}_{1-x-y} \mathrm{Sn}_{x} \mathrm{Mn}_{y}$ Te crystals. Anomalous Hall effect and weak negative magnetoresistance were discovered below $T_{\mathrm{C}}$ due to spin dependent interaction of ferromagnetic clusters with holes. The temperature dependence of thermoelectric power revealed below $T=170 \mathrm{~K}$ a deviation from the linear dependence expected for a semiconductor with a degenerate carrier gas in case of the structural phase transition of ferroelectric type. Investigations should be prolonged for other compositions in order to increase Curie point.

\section{Acknowledgments}

This work was supported in part by the program of the NAS of Ukraine "Nanosystems, nanomaterials, nanotechnologies" Grant No. 85/07-H.

\section{References}

[1] E.I. Rogacheva, O.N. Nashchekina, Y.O. Vekhov, M.S. Dresselhaus, G. Dresselhaus, Thin Solid Films 484, 433 (2005).

[2] Z.M. Dashevsky, L.D. Dudkin, J. Thermoelectr. 1, 93 (1993).

[3] W. Dobrowolski, J. Kossut, T. Story, in: Handbook on Magnetic Materials, vOL. 15, Ed. K.H.J. Buschow, Elsevier-North-Holland, Amsterdam 2003, 289.

[4] A.V. Brodovoy, G.V. Lashkarev, M.V. Radchenko, E.I. Slyn'ko, K.D. Tovstyuk, Fiz. Tekh. Poluprovodn. 18, 1547 (1984).

[5] T. Story, R.R. Galazka, R. Frenkel, P.A. Wolff, Phys. Rev. Lett. 56, 777 (1986).

[6] G.V. Lashkarev, V.I. Sichkovskyi, M.V. Radchenko, V.E. Slynko, E.I. Slynko, T. Story, W. Dobrowolski, W. Knoff, T.Sh. Osmanov, J. Magn. Magn. Mater. 321, 1782 (2009). 